\title{
Electron Microscopic Study of the Transport Mechanism of the Human Placental Villi
}

\author{
By \\ Morio Nakano \\ Department of Obstetrics and Gynecology, Tohoku University, Schro' \\ of Medicine, Sendai; Director: Prof. K. Kushima \\ (Received for publication, November 7,1962 )
}

\section{INTRODUCTION}

The placenta is located between the maternal body and the fetus and is an organ indispensable for the fetus, with the combined functions of endocrine gland, lung, kidney, liver and digestive tract, but the mechanism of these functions is still obscure in many points. In the present study, electronmicroscopic observations were undertaken to study the mode of transition of substances through the normal placenta and the route of transportation of administered substance from the maternal to the fetal side, through the chorionic villi of placenta.

\section{MATERIALS AND METHODS}

Two kinds of placental materials were used: The first series of experiments are on normal placental chorionic villi from gravidae of 2 months through 10 months of pregnancy; and the second series are on those from gravidae of 3 months and 10 months of pregnancy incubated at $37^{\circ} \mathrm{C}$ for $1-2$ hours in Hanks solution containing $10 \%, 20 \%$ or $30 \%$ of Fatgen, a neutral fat emulsion (and in some cases also India ink and colloidal gold). In both series of experiments, placental materials were all immersed in veronal-acetate buffer to correct the $\mathrm{pH}$ value to 7.4 , fixed in isotonic $1 \%$ osmium tetroxide for 1 hour in ice-box, dehydrated with serially diluted ethanol, and embedded with benzoyl-peroxide-added methacrylate for 24 hours at $47^{\circ} \mathrm{C}$. Ultra-thin sections were made from the embedded samples with a JUM type ultra-microtom, and without further staining, or electron-stained with lead hydroxide, phosphotungstic acid and uranyl acetate, were mounted on copper mesh covered with collodion film, were examined under a Hitachi's HU 5 type electron microscope. 


\section{RESULTS}

\section{The Findings of Normal Placentae without Loading}

Numerous microvilli were found of the free surface of the syncytiotrophoblasts, and pinocytotic vesicles were formed in the bases of these microvilli. Vesicles smaller than endoplasmic reticula were scattered all over the syncytiotrophoblasts. Where the cytoplasm projected beyond the free surface, there were vacuoles with varyin. contents and $0.5 \mu$ in diameter, and similar vacuoles were sporadically found near the surface layer of syncytiotrophoblasts (Fig. 1). The endoplasmic reticula were of the rough-surfaced type and were round in the early stage but became flat in the last stage of pregnancy. Their contents unusually consisted of woolly substances of low electron density, but in some, the electron density was higher, so that there were some vacuoles indistinguishable from full-contented vacuoles. Some round homogeneous granules, $1-5 \mu$ in size and of high electron density were found in the syncytiotrophoblasts, probably lipoid granules. Mitochondria and endoplasmic reticula were sometimes found in the course of transition into myelinoid lamellar formations, and it seemed that these formations further changed over into the lipoid granules. Glycogen granules and dense granules, around $50 \mathrm{~m} \mu$ in diameter but of unknown nature, were found in the cytoplasm and the basement membrane. The nucleus of the syncytiotrophoblasts had a double membrane, pores and nucleoli.

There were lacunae of $200-300 \mathrm{~m} \mu$ width at the points where the syncytiotrophoblasts adjoined the cytotrophoblasts. With advance of the fetal age, these lacunae widened cystically, their cytoplasm was entirely of low electron density, the endoplasmic reticula and the RNA granules were few, the former containing only woolly substance but never being full as were some syncytiotrophoblasts (Fig. 3). The syncytiotrophoblasts adjoining the basement membrane were mostly smooth-surfaced in the early stage of pregnancy, but in later months, they began to send out microvillous protrusions toward the basement membrane. Similar protrusions came out from the lower surface of the cytotrophoblasts, too. In some cases, such protrusions penetrated into the basement membrane.

The basement membrane which lies about $20 \mathrm{~m} \mu$ beneath the syncytiotrophoblast and the cytotrophoblast, is about $300 \mathrm{~m} \mu$ thick, showing a membraneous structure, and it is formed by accumulation of fine granular matter of moderate electron density in osmium-tetroxide-fixed specimens (Figs. 2, 4). In the later months of pregnancy, the basement membranes grew in thickness, became less dense in structure and came to contain empty vacancies of $50-100 \mathrm{~m} \mu$ in diameter (Fig. 2-h). Usually, no filament structure could be found in the basement membranes. They contained homogenous dense granules of $100-300 \mathrm{~m} \mu$ in size, of dense fine granules stained darkly by lead hydroxide (Fig. 2-g). As specific formations, 
the basement membrane contained desmosome-like structure (Fig. 2-f) and parts of lamellar structure distinctly formed of 3-4 lamellae in the early stage (Figs. 2-c, 3), cytoplasm of the cytotrophoblasts piercing through the basement membrane in the middle stage, and collagenous fibers stretching down to form filamentous parts in the basement membrane (Fig. 2-d) and lacunae of $2 \mu$ at the widest, in which a homogenous fluid matter was held and finally led to rupture of the basement membrane and release of the fluid into the stroma in the last stage of pregnancy (Figs. 2-j, 5).

The solid matters in the stroma beneath the basement membrane consisted of stroma cells, collagenous fibers and fetal capillaries, which tended to increase with the progress of pregnancy. The remainder of the stroma was filled by woolly fluidal matter of low electron density. In the early stage of pregnancy the stroma cells were small, the collagenous fibers were fine and showed no periodicity and ran in bundles of 5-6 fibers (Fig. 3). In this stage, the stroma cells interconnected through their ramifications and formed cavities, but these contained no transitional cells and were obviously different from capillary lumina. In the middle stage of pregnancy, the formations surrounded by collagenous fibers and possibly identifiable as route of fluids were observed (Fig. 4). In this stage, the stroma cells contained aggregation of fine granules of dense hemosiderin-like substance in some of their endoplasmic reticula, and there were some lamellar formations, probably of lipoprotein in the cells containing homogenous structureless lipoid drops.

The fetal capillaries were often accompanied by pericytes, and the endothelial cells were surrounded by basement membranes $20-40 \mathrm{~m} \mu$ thick. The endothelial cells had no pores, but showed terminal-bar like structure at the part of attachment. Sometimes microvillous protrusions were found stretching towards the inner cavity. The endoplasmic reticula were poorly developed, pinocytotic vesicles being in evidence. The existence of homogenous granulous substance and its release into the capillary could be clearly confirmed. Filament structure was apparent in the endothelial cells (Fig. 6).

\section{The Findings of Incubation in Fat (Fig. 7)}

Tiny Fatgen particles of $0.1-0.3 \mu$ were pinocyted and taken into the syncytiotrophoblasts through the basal part of microvilli, and the fat droplets taken into the endoplasmic reticula of the syncytiotrophoblasts were found enveloped in ergastplasmic membrane and sometimes also in the vacuoles among the other contents of moderate electron density and then making transition into the cytoplasmic ground substance at the surface of syncytiotrophoblasts; such fat droplets had no capsule. The fat droplets, upon reaching the boundary between the syncytiotrophoblasts and cytotrophoblasts, were apparently taken into the cytotrophoblasts rather soon, for fat droplets were only seldom found in the 
space between the layers of two kinds of trophoblasts. The fat particles taken into the cytotrophoblasts were round and around $0.3 \mu$ in diameter (Fig. 8), of high electron density and were contained in the number of above half a dozen per cell.

The fat particles reaching the basement membrane spread diffusely in the membrane. Beneath the basement membrane, too, the particles were no more of droplet shape, except some that retained the round form of $0.5 \mu$ in diameter. Fatgen imported into the stroma formed several droplets in the stroma cells. There were vacuolar distensions of about $0.5 \mu$ in the cytoplasmic protrusions of these cells and fat particles were found in them, too. The fat particles in the stroma cells were uncapsulated and were not contained in the endoplasmic reticula but in the cytoplasmic ground substance. In few cases, fat in granular form was found among the bundle of collagenous fibers of diffuse high electron density, but most part of the fat passed among the collagenous fibers into the pinocytotic vacuoles but more frequently uncapsulated in the cytoplasmic ground substance. Sometimes fat was found pushing up the cell membrane lining the capillary lumen (Fig. 10) in the act of coming and into the lumen, and sometimes fat particles were phagocytosed by leucocytes.

In the case of Fatgen perfused through the umbilical artery, Fatgen particles could be found only in the villous capillary lumina but not in the stroma or the chorionic epithelium.

After incubation in India ink and colloidal gold suspensions, ink or gold particles could be traced as for as the endoplasmic reticula of the chorionic epithelial cells but no further. Nor were they found aggregated to some specified parts.

\section{DISCUSSION}

Since the promontories of the free surface of placenta are provided with microvilli, it may be inferred that they have not only a secretory but also an absorbing function. In the present study, vacuoles of every stage as graded by Bennett ${ }^{1)}$ could be found to suggest one of the processes of matter ingestion, and it was confirmed that such vacuoles play a primary role in the process of placental substance transport, upon experiments of charging fat and other matters. The myelinoid lamellar formation originating in pinocytosis and named "Segresome" by Tanaka'). Arnold et al. ${ }^{3)}$ report on finding tubules running either from the vacuoles or from the basement membrane of the syncytiotrophoblast layer into the intervillous spaces. The present author could not observe such tubules, but in the experiments of charging India ink and Fatgen, he found that the syncytiotrophoblasts readily take in foreign substances into their vacuoles, in the process of matter transportation. Ikawa ${ }^{4}$ and Hashimoto et al. ${ }^{5,6)}$ supposed that the spaces between the syncytiotrophoblast and the cytotrophoblast layers act as route of substance transportation, but no such fact could be demonstrated in 
the present study. Rhodin et $\left.a l_{.}{ }^{7}\right)$ infer that the secretory granules in syncytiotrophoblasts and cytotrophoblasts are produced from the Golgi apparatus, and Wellings et $a l^{8}{ }^{8}$ found milk protein deposits inside the Golgi membrane and is secreted from the cell surface in rat mammary gland, but the present author obtained no findings of Golgi apparatus active in secretion or in forming storage of absorbed substance.

The finding that the basement membrane of the chorionic epithelial cells grows more and more conspicuously with advance of the months of pregnancy, due to the progressive basal infolding and microvillous protrusions formation of the membrane, is presumably one of the morphological features involved in the process of placental substance transportation.

The notion of a basement membrane is a new evolution developed together with the introduction of electron microscopy, but its function and chemical constitution in connection with the process of substance transport and metabolism are little known, as examplified by the notorious obscurity in the properties of renal gromerular basement membrane. Its conformation has been only cursorily described as lamellar and membraneous as viewed under the electron microscope. In the present study, the basement membrane was found to show several morphological changes and that changed substances pass through it in two different manners. Berger ${ }^{9}$ says that the subepithelial basement membrane of the vaginal part of human uterus consists of fine granules and very fine fibers and that these are essentially identical in nature with the granules and collagenous fibers in the epithelial cell membrane, but the present author is inclined to deny the identity in constitution by considering the difference in the results of electron staining.

The stroma cells contained vacuoles including hemosiderin-like granules of high electron density, lipoid droplets and lamellar formations, and after incubation in fat showed increase of fat in them and held fat at the cytoplasmic ramification; these findings suggest that some substances are transported through the stroma cells. Outside the stroma cells there were found extravascular fluid routes lined by collagenous fibers, and some of the charged substance was found among the collagenous fibers. Most part of the charged fat, however, was found within the stroma cell cytoplasm and only a small part of it outside the cells. It may be inferred that the substance imported into the pericytes through or along the stroma cells is forwarded into the endothelial cytoplasm through rupture of the basement membrane of the capillaries and finally flows into the capillary lumen. Palay et $a l .{ }^{10,11)}$, upon electron-microscopical examination of rat small intestine, found that fat is carried through the intercellular route since its first intake by the endoplasmic reticula of the epithelial cells till its expulsion into the intercellular spaces of epithelial cells and thence through the extracellular route, first being heaped up on the basement membrane and finally being discharged into the 
lumph duct lumen.

The findings in the present study seem to indicate that in the placental chorionic villi most of the fat particles take the intracellular route, differing from the findings in the small intestine. Okuda ${ }^{12,13)}$ has reported on his histochemical study on the subject, in which he found a small quantity of charged fat transported to the fetal side through rat placenta and upon incubation also through human placenta. By similar method, the present author confirmed electron-microscopically that fat in its intact constitution passes through the syncytiotrophoblasts into the fetal capillaries.

\section{SUMMARY}

The feto-maternal transportation of substances through the villi of human placentae from gravidae of 2 through 10 months of pregnancy and human placenta charged with fat in vitro was electron-microscopically studied.

The following conclusions in summary were arrived at:

1) Promontory-like cytoplasmic protrusion apparently partaking in the processes of feto-maternal substance transportation through and secretory function of the placenta, and many microvilli related with pinocytosis were found on the free surface of the layer of syncytiotrophoblasts. The endoplasmic reticula gradually changed into route of substance transportation and the surface of basement membrane of syncytiotrophoblasts and cytotrophoblasts became more and more conspicuously marked by basal infoldings and microvilli-like protrusion, with the advance of months of pregnancy.

2) The basement membrane is usually a homogenous structure formed by aggregation of fine granulous substance and was found to grow thicker with fetal

age. It showed soundly morphological changes and included some matter indicative of substance transportation through it.

3) The stromal collagenous fibers increased both in number and in size with progress of pregnancy and were found to form a part of fluid route through the stroma.

4) The fetal capillaries had a basement membrane, their lumen enlarged and their endothelium thined from month to month. Here, the main substance transportation was apparently effected through pinocytosis.

5) Findings were obtained that fat particles are taken in by syncytiotrophoblasts from through pinocytosis. A part of the fat particles pass into cytotrophoblasts from syncytiotrophoblasts. The fat particles reaching the basement membrane beneath the chorionic epithelium permeate the membrane in diffuse form, but a part of them were seen going through the membrane in unchanged particle shape.

6) The fat transportation in stroma was found mainly passing through the cytoplasm of stroma cells from the chorionic villus side to the fetal capillary 
side. Besides, some fat particles were found extracellularly. Fat taken up by the perivascular pericytes was observed slowly passing over into the endothelial cells of the fetal-side capillaries, and through the cells finally to flow into the capillary blood through ruptures in the cell membrane.

\section{References}

1) Bennett, S., J. Biophysic. \& Biochem. Cytol., 1956, 2 Suppl., 99.

2) Tanaka, H., Saishin Igaku (Jap.), 1962, 17, 206.

3) Arnold, M. et al., Arch. Gynäk., 1961, 196, 238.

4) Ikawa, A., J. Jap. Obst. \& Gynec. Soc., 1959, 6, 219.

5) Hashimoto, M. et al., ibid., 1960, 7, 44.

6) Hashimoto, M. et al., ibid., 1960, 7, 122.

7) Rhodin, J. et al., J. Ultrastructure Research, 1962, 6, 88.

8) Wellings, R. et al., J. Biophisic. \& Biochem. Cytol., 1961, 9, 479.

9) Berger, J., Gyneacologia, 1961, 152, 208.

10) Palay, S. et al., J. Biophysic. \& Biochem. Cytol., 1959, 5, 363.

II) Palay, S. et al., ibid., 1959, 5, 373.

12) Okuda, Y., J. Jap. Obst. \& Gynec. Soc. (Jap.), 1959, 11, 899.

13) Okuda, Y., Tohoku J. Exper. Med., 1960, 72, 290.

14) Yamaguchi, R. et al., J. Jap. Obst. \& Gynec. Soc., 1960, 7, 35.

Abbreviations in the Figures.

$\begin{array}{lll}\text { Tv. sp. } & : & \text { intervillous space } \\ \text { Sy. } & : & \text { syncytiotrophoblast } \\ \text { Cy. } & : & \text { cytotrophoblast } \\ \text { N. } & : & \text { nucleus } \\ \text { B.m. } & : & \text { basement membrane } \\ \text { Str. } & : & \text { stroma } \\ \text { Str.c. } & : & \text { stroma cell } \\ \text { Clg. } & : & \text { collagenous fiber } \\ \text { P. } & : & \text { pericytes } \\ \text { Et. } & : & \text { capillary endothelium. } \\ \text { M.f. } & : & \text { microfibril } \\ \text { F.c. } & : & \text { fetal capillary } \\ \text { V. } & : & \text { vacuole }\end{array}$

All scales show $1 \mu$, and arrows show fat droplets. 


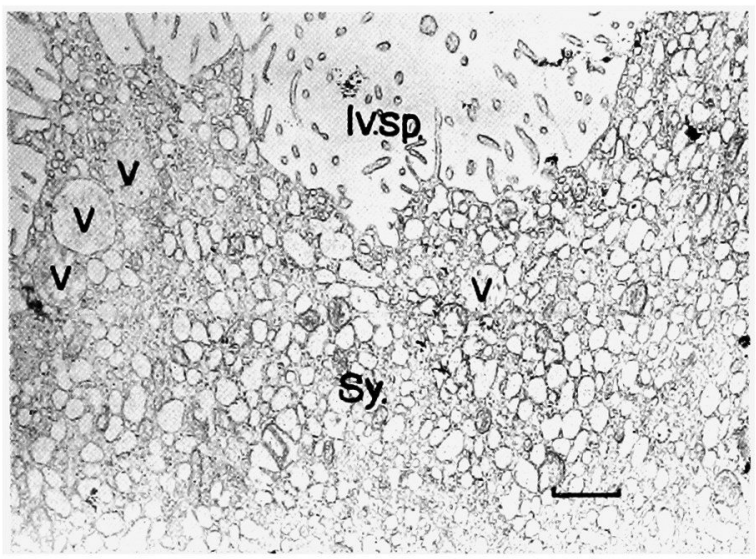

Fig. 1. Free surface of syncytiotrophoblast at 3 months of pregnancy. Many contents in the vacuoles and numerous pinocytotic vesicles are recognized.

a)

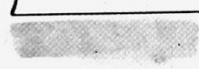

b)

c)

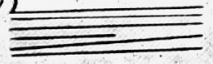

d)

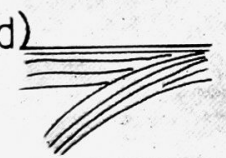

e)

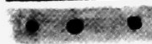

f)

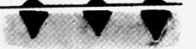

i)

n)
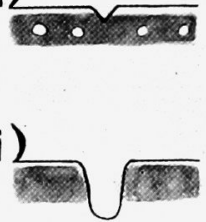

g)

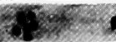

j)

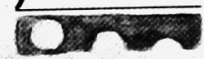

Fig. 2. Models of basement membrane of the chorionepithelium. 


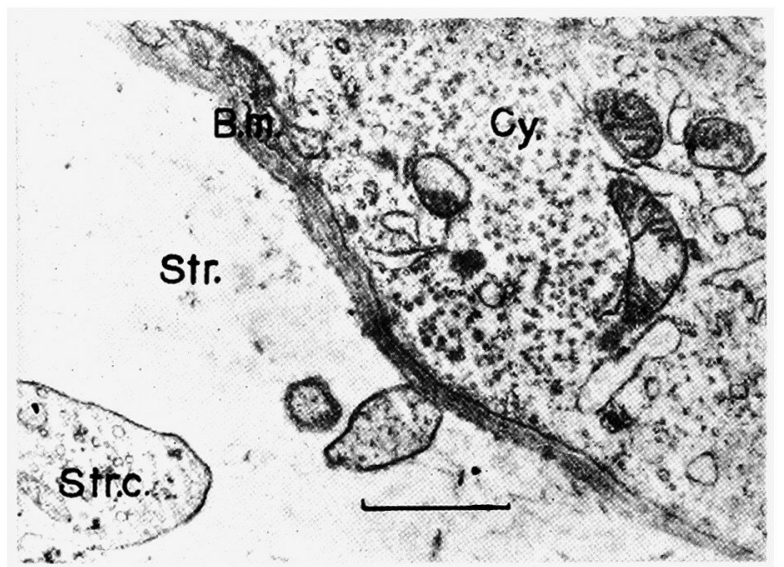

Fig. 3. Cytotrophoblast, basement membrane and stroma cells at the first trimester of pregnancy. Basement membrane consists of three or four layers.

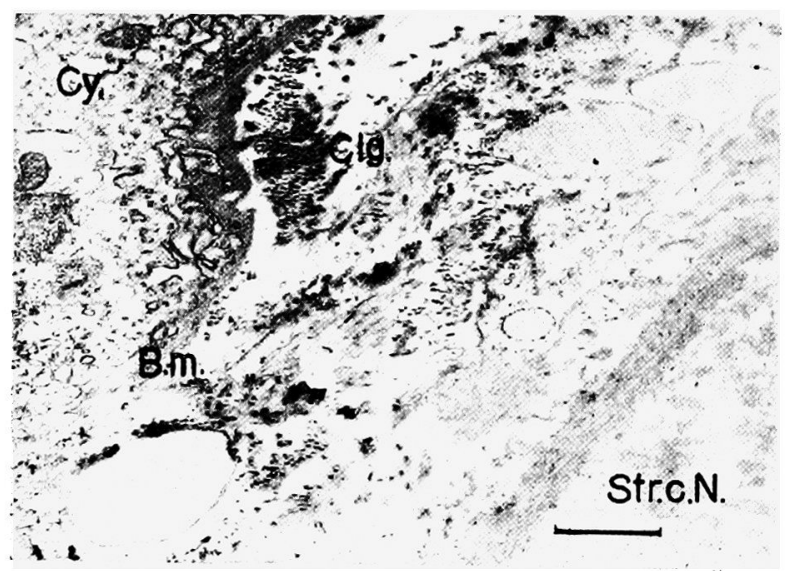

Fig. 4. Cytotrophoblast, basement membrane, collagenous fibers and stroma cells. Extra-cellular routes of fluid transport (?) at the middle trimester of pregnancy. 


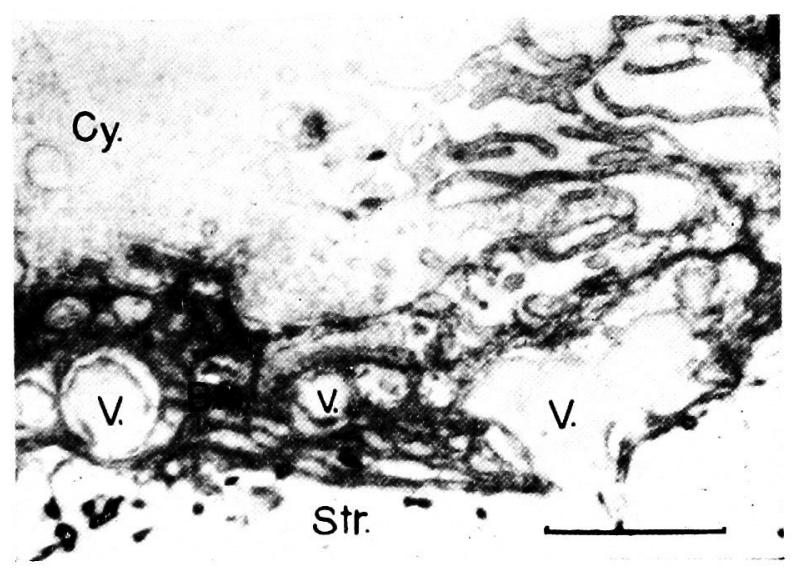

Fig. 5. Cytotrophoblast has many microvilli. This basement membrane contains fluidal substance which finally leads to rupture to stroma.

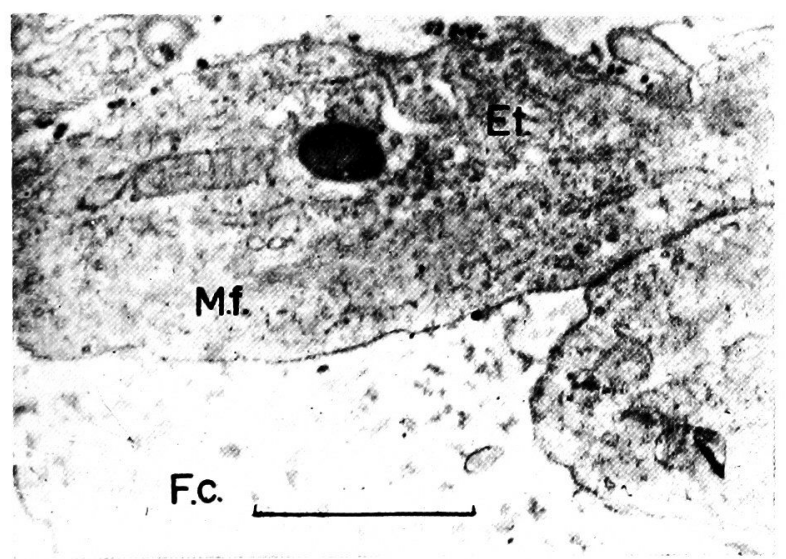

Fig. 6. Endothelium of fetal capillary. It contains mitochondria, round dense body and microfilament. 


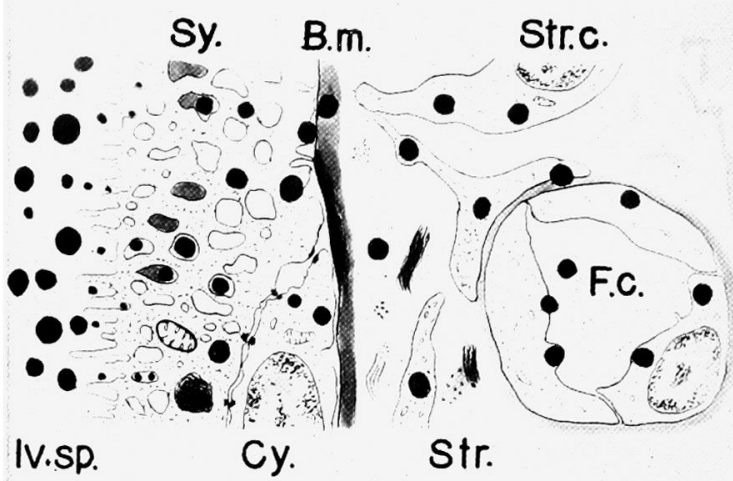

Fig. 7. A model on fat droplet transport mechanism from inter-villous space to fetal capillary.

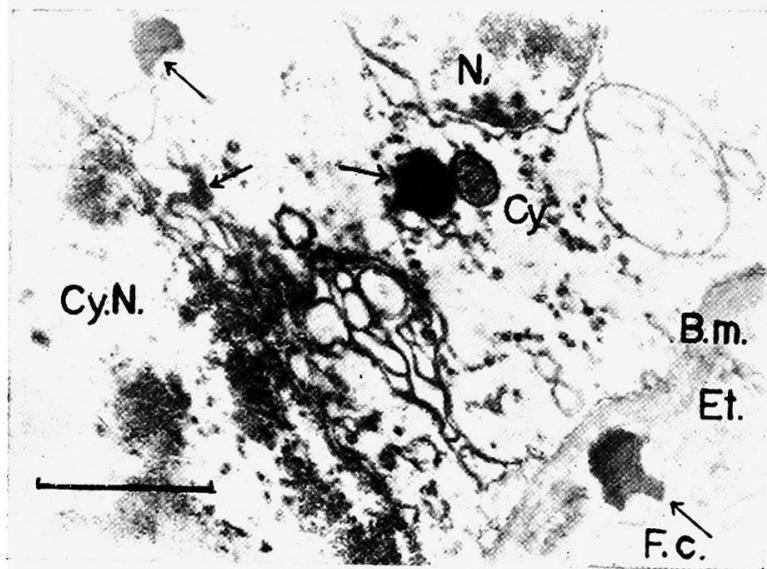

Fig. 8. Fat droplets in cytotrophoblasts, inter-cellular space and in fetal capillary endothelium. 


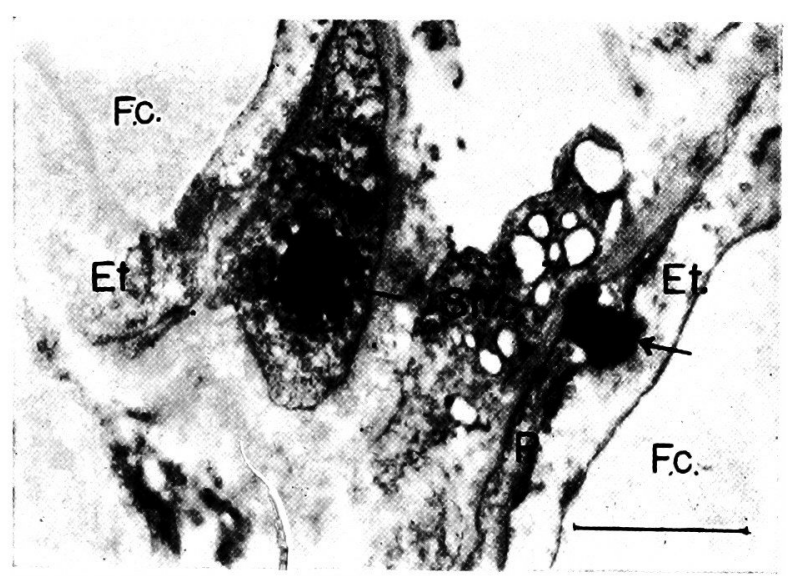

Fig. 9. Two fat droplets are in pericytes. Right drop is going to penetrate into endothelial cytoplasm.

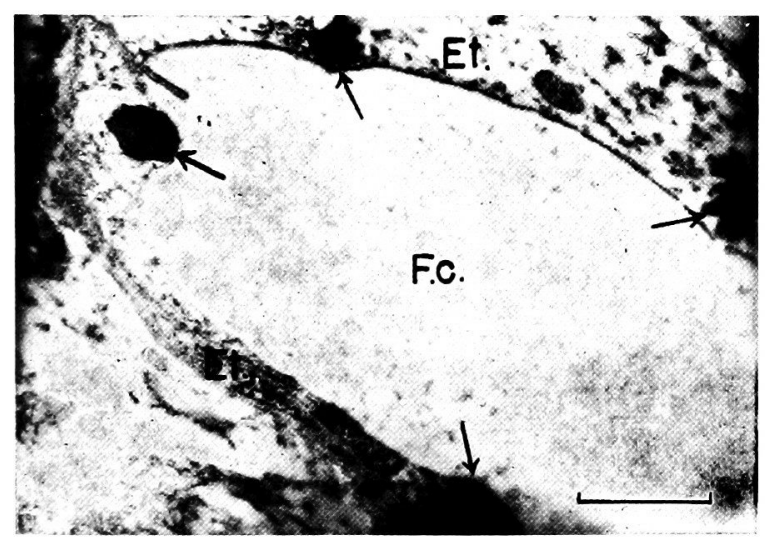

Fig. 10. Four fat droplets are going to be sprung into capillary cavity from endothelial cytoplasm. 